\title{
Clasificación pentadal de vientos frente a la costa Peruana
}

\author{
David Correa $^{* 1,2}$, Adolfo Chamorro ${ }^{1}$, Jorge Tam ${ }^{2}$ \\ ${ }^{1}$ Instituto del Mar del Perú, Callao Perú \\ ${ }^{2}$ Grupo de Investigación de Mecánica de Fluidos, UNMSM, Perú
}

Recibido 06 julio 2020 - Aceptado 13 diciembre 2020

\begin{abstract}
Resumen
El viento frente a la costa peruana tiene influencia en los procesos físicos, químicos y biológicos marinos, tanto en las capas superficiales como sub-superficiales e impacta sobre las corrientes marinas costeras, la temperatura superficial del mar, la capa de mezcla vertical, el transporte y retención de larvas en el mar. Así mismo, tiene influencia en el acercamiento de aguas oceánicas hacia la costa y en otros casos favorece la extensión de Aguas Costeras Frías y Aguas de Mezcla frente a la costa peruana mediante el afloramiento costero. Por tanto, los eventos de intensificación y debilitamiento del viento costero tienen una fuerte repercusión sobre las condiciones ambientales y el ecosistema. En este trabajo, se utilizaron datos de vientos satelitales obtenidos a través del dispersometro ASCAT (Advanced Scatterometer) sobre la franja costera de $100 \mathrm{~km}$ (aproximadamente 62 millas náuticas) frente a Perú, en cinco zonas: Paita, Chicama, Callao, San Juan de Marcona e Ilo, entre el periodo del 21 de marzo del 2007 al 03 de marzo del 2019, para analizar la variabilidad intraestacional del viento y determinar, basado en el agrupamiento de cuartiles y promedios móviles, las intensidades mínimas y máximas del viento para la identificación de eventos de intensificación o debilitamiento respectivamente. Los resultados muestran que la variabilidad intraestacional del viento es menor en la zona norte (Paita y Chicama), que en la zona centro-sur (Callao, San Juan de Marcona e Ilo), que presenta una mayor variabilidad. Las intensidades mínimas y máximas pentadales para identificar eventos de intensificación y debilitamiento del viento muestran diversos rangos de variación a lo largo del año y ubicación a lo largo de la costa peruana. Dependiendo de la estación del año, Ilo presenta intensidades mínimas de viento que varían entre 2,1 a 3,8 ms-1 (debilitamiento) y máximas entre 4,4 a 5,2 ms-1 (intensificación); en cuanto que Paita, Callao y Chicama, presentan intensidades mínimas de vientos que varían entre 2,3 a 4,8 ms-1 (debilitamiento) y máximas de 4,2 a 7,3 ms-1 (intensificación); en cuanto que San Juan de Marcona presenta intensidades mínimas de vientos entre 3,7 a 6,6 ms-1 (debilitamiento) y máximas de 5,6 a 9,5 ms-1 (intensificación). Finalmente se determinó la cantidad de días de intensificación y debilitamiento del viento por año, encontrándose predominancia de eventos de intensificación de vientos durante los años 2007, 2010 y 2013, y predominancia de eventos de debilitamiento de vientos durante los años 2012, 2013, 2016 y 2017.
\end{abstract}

Palabras claves: Vientos, clasificación, cuartil inferior, cuartil superior, costa peruana.

\section{Pentadal classification of wind off Peruvian coast}

\begin{abstract}
The wind off the Peru coast has influence on marine physical, chemical and biological processes, both in surface and sub-surface layers and impacts coastal sea currents, sea surface temperature, vertical mixing layer, transport and retention of larvae in the sea. Furthermore it influences the approach of oceanic waters towards the coast and in other cases it favors the extension of Cold Coastal Waters and Mixing Waters off the Peruvian coast through the coastal upwelling. Therefore, the intensification and weakening of coastal wind have a strong impact on environmental conditions and the ecosystem. In this work, satellite wind data obtained through the ASCAT (Advanced Scatterometer) scaterometer from a coastal strip up to $100 \mathrm{~km}$ (approximately 62 nautical miles) off Peru was used in five areas: Paita, Chicama, Callao, San Juan de Marcona and Ilo, during the period from March 21, 2007 to March 03, 2019, in order to analyze the intraseasonal variability of the wind and determine, based on the grouping of quartiles and moving averages, the

dcorrea@imarpe.gob.pe
\end{abstract}


Rev. Inv. Fís. 23(3), (2020)

minimum and maximum wind intensities for the identification of events of intensification or weakening respectively. The results show that the intra-seasonal wind variability is lower in the northern zone (Paita and Chicama), than in the central-southern zone (Callao, San Juan de Marcona and Ilo), which presents greater variability. The minimum and maximum pentadal intensities to identify wind intensification and weakening events showed different ranges of variation throughout the year and location along the Peruvian coast. Depending on the season, Ilo has minima wind intensities that vary between 2.1 to $3.8 \mathrm{~ms}-1$ (weakening) and maxima between 4.4 to $5.2 \mathrm{~ms}-1$ (intensification); while Paita, Callao and Chicama, have minimum wind intensities that vary between 2.3 to $4.8 \mathrm{~ms}-1$ (weakening) and maxima between 4.2 to 7.3 ms-1 (intensification); while San Juan de Marcona has minimal wind intensities between 3.7 to $6.6 \mathrm{~ms}-1$ (weakening) and maxima between 5.6 to $9.5 \mathrm{~ms}-1$ (intensification). Finally, the number of days per year of wind intensification and weakening was determined, resulting in a predominance of wind intensification events during the years 2007, 2010 and 2013, and a predominance of wind weakening events during the years 2012, 2013, 2016 and 2017.

Keywords: Winds, classification, lower quartile, upper quartile, and Peruvian coast.

\section{Introducción}

El viento como parte de la interacción atmosfera océano tiene una gran influencia sobre los procesos físicos, químicos y biológicos que ocurren en la capa superficial y sub-superficial del mar peruano, principalmente asociados a la dinámica del afloramiento costero, impactando sobre las corrientes marinas superficiales locales, la temperatura del mar, la profundidad de la capa mezcla, la suspensión, retención y transporte de larvas, huevos y nutrientes.

El viento dentro de su variación intraestacional presenta eventos de intensificación y debilitamiento, siendo estos variables en la cantidad de días de duración, en velocidades promedio y máxima que alcanzan, así como en la extensión espacial que abarcan, dependiendo también de la época del año en que ocurren. Existen estudios como (Cury \& Roy, 1989), que relacionan el transporte Ekman y la turbulencia del viento con la sobrevivencia, reproducción y reclutamiento de los peces pelágicos en el sistema de afloramiento de borde Este (Perú, California, Senegal, entre otros).

En este sentido, se analiza la variabilidad del viento a lo largo de la costa peruana, a través de la metodología de agrupamiento de cuartiles, llegándose a determinar eventos de intensificación y debilitamiento de viento. Los eventos se definen como la ocurrencia de vientos que mantienen velocidades sobre o por debajo de las intensidades mínimas de viento por tres o más días consecutivos.

Los eventos de intensificación de viento, en su mayoría se relacionan con la ocurrencia de procesos de afloramiento de aguas sub superficiales, reflejándose en la expansión espacial de las Aguas Costeras Frías y Aguas de Mezcla frente a la costa peruana, así la aparición de condiciones de hipoxia; mientras que los eventos de debilitamiento de viento, en su mayoría se relacionan con el acercamiento de Aguas Tropicales y Aguas Ecuatoriales Superficiales (principalmente en la zona norte del Perú) y Aguas Subtropicales Superficiales a las zonas costeras de Perú. Por otro lado eventos de vientos débiles durante los meses de primavera y verano, se relacionan con la ocurrencia de ondas de calor y eventos El Niño Costero.

\section{Material y Métodos}

Los vientos satelitales son adquiridos a través de los dispersómetros ASCAT, a bordo de los satélites del programa de satélites Meteorológicos Operativos (MetOp), de la Organización Europea para la Explotación de Satélites Meteorológicos (EUMETSAT). La información fue obtenida del Centre de Recherche et d'Exploitation Satellitaire (CERSAT), en L'Institut Français de Recherche pour l'Exploitation de la Mer (IFREMER) de Francia, ftp://ftp.ifremer.fr/ifremer/cersat/products/gridded/mwf-ascat.

Los satélites MetOp llevan un conjunto de instrumentos que miden diversos parámetros atmosféricos a nivel global, entre ellos la velocidad y dirección del viento sobre la superficie de los océanos, las medidas son equivalentes a una altura promedio de 10 metros sobre el nivel del mar, sobre una resolución espacial de $0.25^{\circ}$ (aproximadamente $27.5 \mathrm{~km}$ ) en longitud y latitud. Para conocer sobre los datos, metodologías, algoritmos de cálculo de las componentes zonal y meridional del viento satelital, se recomienda revisar (Bentamy, Katsaros, Drennan, \& Forde, 2002) y (Bentamy \& Croizé-Fillon, 2012)

El área de estudio, comprende cinco zonas (Paita, Chicama, Callao, San Juan de Marcona e Ilo) a lo largo y dentro de $100 \mathrm{~km}$ (62 millas náuticas) frente de la costa peruana; temporalmente se analiza la información de vientos satelitales ASCAT entre el 21 de marzo del 2007 y el 03 de marzo del 2019.

Para el cálculo de la varianza y el promedio del viento en las cinco zonas, se analizó la información a través de pentadas (5 días) multianuales, calculadas desde enero a diciembre, en cada pentada se incluyó información de vientos de quince días antes y después de la pentada en evaluación (ventana móvil de 30 días), Figura 1a. El cálculo de las intensidades mínimas de debilitamiento e intensificación se realizaron sobre cada pentada, a través de la metodología de agrupamiento por cuartiles, Figura $1 \mathbf{b}$

Finalmente se calculó el número de eventos de debilitamiento e intensificación de vientos por año, basado en el criterio de que el viento presente velocidades sobre o 
Rev. Inv. Fís. 23(3), (2020)

por debajo las intensidades mínimas de intensificación o debilitamiento de viento por tres o más días consecutivos.

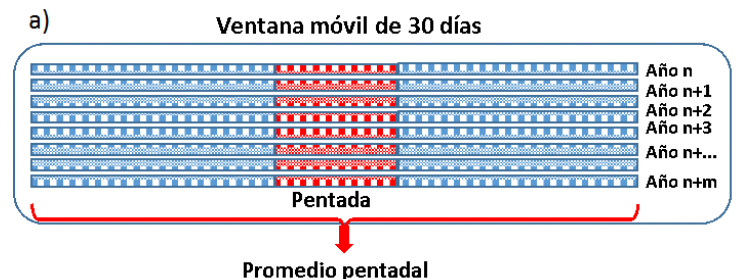
cuartil superior

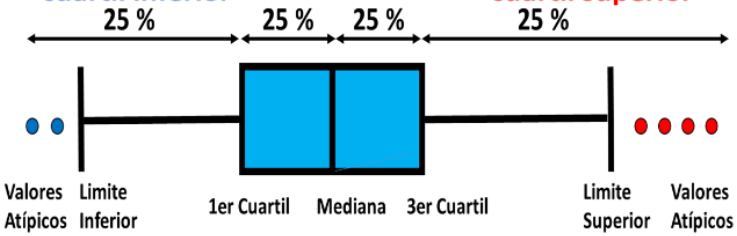

Figura 1: a) Esquema de selección de información a través de pentadas con medias móviles de 30 días, b) agrupamiento de información para el cálculos de vientos bajo cuartil inferior y sobre cuartil superior

\section{Resultados y discusión}

\section{Variabilidad del viento}

El análisis de la varianza de los vientos entre los años 2007 y 2019 sobre las cinco localizaciones, muestran comportamientos diferenciados, por un lado se tiene que Paita y Chicama, presentan una menor variabilidad de vientos, mientras que Callao, San Juan de Marcona e Ilo, presentan una mayor variabilidad, principalmente sobre los meses de mayo a setiembre. La menor variabilidad de viento es de 1,2 ms-1 (noviembre, Chicama), y la mayor es de 6,3 ms-1 (julio y setiembre, San Juan de Marcona).

En general, las menores varianzas se presentaron entre los meses de enero a abril y octubre a diciembre, oscilando entre 1,2 ms-1 (noviembre, Chicama) a 2,8 ms-1 (octubre, San Juan de Marcona), a excepción de Paita y Chicama que presentaron una varianza inferior a 2,5 ms-1 (abril, Paita) durante todo el año.

Por otro lado las mayores varianzas se presentaron entre los meses de mayo a setiembre, diferenciándose dos rangos: para Callao e Ilo la variabilidad del viento osciló entre 2,4 ms-1 (setiembre, Ilo) a 4,0 ms-1 (agosto, Callao), mientras que para San Juan de Marcona, la variabilidad del viento osciló entre 3,5 ms-1 (mayo y setiembre) a 6,3 ms-1 (julio y agosto) (Figura 2).

Finalmente el análisis de la varibilidad de los vientos, coincide con (Dewitte, y otros, 2011) en demostrar que la variabilidad intra estacional del viento en la zona centro sur (Callao y San Juan de Marcona) frente a Perú están relacionados a procesos estacionales de escala regional asociados a la dinámica del Anticiclón del Pacifico Sur.

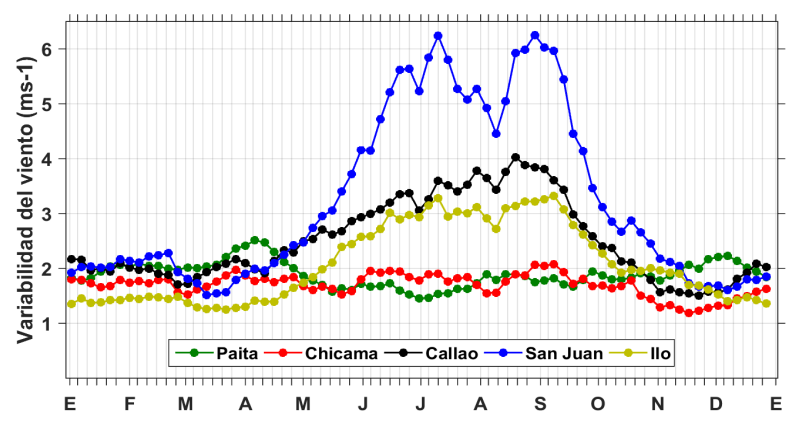

Figura 2: a) Cuartiles superiores y b) Cuartiles inferiores de viento a lo largo y dentro de la franja de $100 \mathrm{~km}$ frente a la costa peruana

\section{Vientos bajo cuartil inferior y sobre cuartil superior}

Los promedios de las intensidades mínimas de viento fueron calculados sobre cada pentada (cinco días) en un ciclo anual promedio, a través de la metodología de agrupamiento por cuartiles, Los vientos se clasificaron en dos tipos: (i) viento que se encuentren por debajo del primer rango inter-cuartil histórico $(25 \%$ de datos de vientos), y (ii) viento que se encontraron sobre el tercer rango inter-cuartil histórico (75\% de datos de vientos).

En la Figura 3 se observa que los cuartiles inferiores varían entre 2,0 ms-1 (agosto, Ilo) a 6,6 ms-1 (julio, San Juan de Marcona), Paita, Callao y Chicama, presentan cuartiles inferiores que varían entre 2,3 a 4,8 ms-1, mientras que San Juan de Marcona presenta cuartiles inferiores entre 3,7 a 6,6 ms-1 e Ilo que varía entre 2,1 a $3,8 \mathrm{~ms}-1$. Respecto a los cuartiles superiores, varían entre 4,2 a 9,5 ms-1, de lo cual San Juan de Marcona presenta cuartiles superiores entre 5,6 a 9,5 ms-1 e Ilo que presenta cuartiles superiores más bajos, entre 4,4 a 5,2 ms-1.

Teniendo en cuenta la propuesta de escala de vientos para la zona costera y oceánica frente a Perú, definida en (Correa et al., 2018), como vientos débiles (< 4,1 ms-1), vientos moderados (entre 4,1 a $6,8 \mathrm{~ms}-1)$ y fuertes $(>6,8$ ms-1). Ilo tendría un rango de vientos normales históricos que oscilaría entre 3,8 a 5,2 ms-1, (vientos débiles a moderados), mientras que Paita, Chicama y Callao, presentarían vientos débiles a moderadas durante los meses enero a abril y noviembre a diciembre, y vientos moderados a fuertes durante los meses de mayo a noviembre; en el caso de San Juan de Marcona, presentaría vientos moderados durante enero a marzo, y moderados a fuertes durante abril a diciembre, Figura 3.

Finalmente se tiene que durante los meses de invierno y primavera predominan mayores velocidades de vientos asociados al acercamiento o intensificación del Anticiclón del Pacifico Sur hacia las costas de Suramérica, mientras que las menores velocidades del viento se dan en los meses de verano asociado al posicionamiento estacional en el hemisferio sur de la Zona de Convergencia Inter-Tropical 
Rev. Inv. Fís. 23(3), (2020)
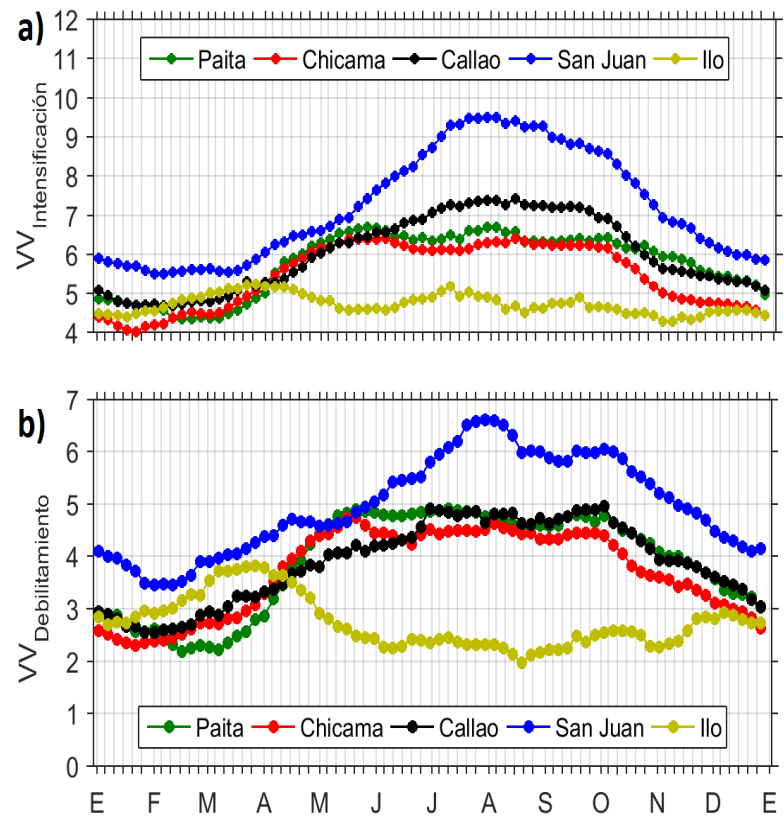

Figura 3: a) Esquema de selección de información a través de pentadas con medias móviles de 30 días, b) agrupamiento de información para el cálculos de vientos bajo cuartil inferior y sobre cuartil superior.

\section{Evolución temporal de tipos de vientos}

En la Tabla 1, se muestra la cantidad de días por año de vientos bajo el cuartil inferior y sobre el cuartil superior para las cinco zonas a lo largo de la costa peruana. De lo cual se tiene un máximo de 107 días (Chicama, 2010) y un mínimo de 3 días (Chicama, 2012) de vientos sobre el cuartil superior y un máximo de 82 días (Paita, 2016) y un mínimo de 13 días (Chicama, 2010 e Ilo, 2008) de vientos por debajo el cuartil inferior de vientos. No se considera en el análisis el año 2007 por contar con solo 286 días.

La mayor cantidad de días de vientos bajo el cuartil inferior se produjeron durante los años 2012, 2013, 2016 y 2017, llegando a alcanzar hasta 82 y 81 (Paita, 2016 y San Juan de Marcona, 2012, respectivamente) y 71 días (Chicama, 2012 y Callao, 2013). Mientras que, la mayor cantidad de días de vientos sobre el cuartil superior se produjeron durante los años 2010 y 2015, llegando a alcanzar hasta 107 (Chicama, 2010) y 90 días (Paita, 2015)

Finalmente, la ocurrencia de más de 50 días de vientos bajo el cuartil inferior entre San Juan de Marcona y Paita en el año 2017, que ocurrió principalmente durante los meses de verano, dieron lugar a un rápido y notable incremento de la temperatura superficial del mar, , iniciando El Niño Costero 2017 (Garreaud, 2018; Echevin, y otros, 2018; Escudero, Alburqueque, \& Xu, 2019). Por otro lado, la ocurrencia de más de 60 días de vientos sobre el cuartil superior entre San Juan de Marcona y Paita en los años 2010 y 2015 estarían asociados a la intensificación del viento costero frente a Perú durante eventos El Niño típico (Enfield, 1981; Kessler, 2006; Chamorro, y otros, 2018).

Tabla 1: Número de días con vientos sobre el cuartil superior y bajo el cuartil inferior por año a lo largo y dentro de la franja de $100 \mathrm{~km}$ frente a Paita, Chicama, Callao, San Juan de Marcona

\begin{tabular}{ccccccc}
\hline Año & Paita & Chicama & Callao & San Juan & Ilo & $=$ Promedio \\
\hline $2007 * *$ & $55(40) *$ & $63(11)$ & $78(10)$ & $60(19)$ & $47(16)$ & $61(19)$ \\
2008 & $63(26)$ & $43(51)$ & $45(29)$ & $36(48)$ & $69(13)$ & $51(33)$ \\
2009 & $48(46)$ & $35(49)$ & $43(39)$ & $71(36)$ & $49(50)$ & $49(44)$ \\
2010 & $72(34)$ & $107(13)$ & $74(35)$ & $67(38)$ & $28(39)$ & $70(32)$ \\
2011 & $89(33)$ & $41(26)$ & $37(44)$ & $25(59)$ & $39(46)$ & $46(42)$ \\
2012 & $19(55)$ & $3(71)$ & $24(56)$ & $34(81)$ & $44(56)$ & $25(64)$ \\
2013 & $62(23)$ & $15(48)$ & $21(71)$ & $21(65)$ & $24(49)$ & $29(51)$ \\
2014 & $45(31)$ & $25(34)$ & $69(31)$ & $67(34)$ & $47(47)$ & $51(35)$ \\
2015 & $90(36)$ & $69(44)$ & $62(36)$ & $71(61)$ & $38(49)$ & $66(45)$ \\
2016 & $13(82)$ & $10(38)$ & $41(57)$ & $35(55)$ & $27(31)$ & $25(55)$ \\
2017 & $40(76)$ & $48(59)$ & $42(62)$ & $39(56)$ & $34(58)$ & $41(62)$ \\
2018 & $57(30)$ & $31(61)$ & $22(65)$ & $38(33)$ & $29(55)$ & $35(49)$ \\
\hline \multicolumn{7}{r}{ Sin paréntesis se representa la cantidad de días de vientos sobre el cuartil superior por año y en paréntesis }
\end{tabular}


Rev. Inv. Fís. 23(3), (2020)

\section{Conclusiones}

La variabilidad del viento frente y dentro de los $100 \mathrm{~km}$ de la costa peruana, muestran diferencias entre la zona norte (Paita y Chicama), con menor variabilidad de viento, respecto la zona centro sur (Callao, San Juan de Marcona e Ilo), que presenta una mayor variabilidad. Esta diferencia se debería a que la zona centro-sur de la costa peruana tendría mayor influencia del forzamiento remoto regional asociado al Anticiclón del Pacifico Sur.

Los cuartiles pentadales superior e inferior de vientos muestran diversos rangos de variación a lo largo del año y ubicación a lo largo de la costa peruana, diferenciándose Ilo, que presenta una menor rango de variación inter cuartil histórica, frente a Paita, Callao, Chicama, y San Juan de Marcona que presentan un mayor rango de variación inter cuartil histórica, con una oscilación estacional.

El análisis del número de días de vientos sobre el cuartil superior o por debajo el cuartiles inferior por año, muestra que los años 2007, 2010 y 2015, presentaron mayor cantidad vientos sobre el cuartil superior, con una cantidad promedio entre 61 a 70 días, mientras que los años 2012, 2013 y 2016 presentaron una menor cantidad de vientos sobre el cuartil superior, con una cantidad promedio de 25 a 29 días. Por otro lado, la mayor cantidad de días de vientos bajo el cuartil inferior, ocurrieron durante los años 2012, 2013, 2016 y 2017, con una cantidad promedio entre 51 a 64 días.

\section{Referencias}

Bentamy, A., \& Croizé-Fillon, D. C. (2012). Gridded surface wind fields from Metop/ASCAT measurements. International journal of remote sensing, 33(6), 1729-1754.

Bentamy, A., Katsaros, K., Drennan, W. M., \& Forde, E. B. (2002). Daily surface wind fields produced by merged satellite data. $A G U$ Geophysical Monograph Series, 127, 343-349.

Chamorro, A., Echevin, V., Colas, F., Oerder, V., Tam, J., \& Quispe-Ccalluari, C. (2018). Mechanisms of the intensifications of the upwelling-favorable winds durind El Niño 1997-1998 in the Peruvian upwelling system. Clim. Dynamic, 1-17. doi:10.1007/s00382-018-4106-6

Correa, D., Vásquez, L. \& Gutiérrez, D. (2018). Propuesta de escala de vientos para la zona costera y oceánica frente a Perú. Informe interno, IMARPE, Callao.

Cury, P., \& Roy, C. (1989). Optimal envirommental windows and pelagic fish recruitment success in upwelling areas. Canadian Journal of Fisheries and Aquatic Sciences, 46(4), 670-680.

Dewitte, B., Illig, S., Renault, L., Goubanova, K., Takahashi, K. (2011). Modes of covariability between sea surface temperature and wind stress intraseasonal anomalies along the coast of Peru from satellite observations (2000-2008). JGR, vol 116. doi:10.1029/2010JC006495
Echevin V, Colas F, Espinoza-Morriberon D, Vasquez L, Anculle T and Gutierrez D (2018).

Forcings and Evolution of the 2017 Coastal El Niño Off Northern Peru and Ecuador. Front. Mar. Sci. 5:367. doi: 10.3389/fmars.2018.00367

Enfield D. (1981). Thermally-driven wind variability in the planetary boundary layer above Lima, Peru. J. Geophys Res 86(C3): 2005-2016.

Garreaud, R. D. (2018). A plausible atmospheric trigger for the 2017 coastal El Niño. Int. J. Climatol. 38, e1296-e1302. doi: 10.1002/joc.5426

Kessler, W. S. 2006. The circulation of the eastern tropical Pacific: A review, Prog. Oceanogr., 69(2-4), 181-217, doi:10.1016/ j.pocean.2006.03.009.

Paulino, C., Escudero, L., Alburqueque, E., \& Xu, H. (2019). Características de El Niño Costero 2017 mediante observación satelital. Boletín del IMARPE, 34(1), 91-104. Obtenido de http://biblioimarpe.imarpe.gob.pe/bitstream/1234 56789/3359/1/Boletin\%2034\%281\%29-8.pdf 